\title{
Probing the receptor recognition site of the FimH adhesin by fimbriae-displayed FimH-FocH hybrids
}

\author{
Thomas B. Knudsen and Per Klemm \\ Author for correspondence: Per Klemm. Tel: +4545252506 . Fax: +4545932809 . \\ e-mail: pk@im.dtu.dk
}

Department of Microbiology, Building 301, Technical University of Denmark, DK-2800 Lyngby, Denmark

\begin{abstract}
Type 1 fimbriae are surface organelles of Escherichia coll which mediate Dmannose-sensitive binding to different host surfaces. This binding is conferred by the minor fimbrial component FimH. The binding domain of the FimH adhesin has been studied by constructing hybrids of FimH and a homologous protein, Foch, originating from F1C fimbriae. F1C fimbriae do not bind to Dmannosides or confer agglutination of any known types of erythrocytes or yeast. It was previously shown that the FocH protein can be readily substituted by the FimH adhesin, resulting in hybrid fimbriae with the same binding characteristics as type 1 fimbriae. The receptor binding of fimbriae-presented chimeric FimH-FocH hybrids was studied. FimH-Foch fusions encompassing $72 \%$ of the $\mathrm{N}$-terminus of FimH fused to the complementary sector of FocH conferred agglutination of erythrocytes and yeast cells at a comparable level to FimH. Surprisingly, it was also found that similar fusions containing between 56 and $66 \%$ FimH still conferred binding to yeast cells, Dmannose-BSA and D-mannose-beads but did not give rise to agglutination. The receptor binding capacity of fusions containing $\mathbf{5 0 \%}$ or less of the FimH Nterminal region was virtually abolished. The results point to the presence of a D-mannose-receptor-binding core domain in FimH, the affinity of which is modulated by other sectors of the protein to enable binding to extended mannose-containing targets.
\end{abstract}

Keywords: adhesin chimeras, bacterial adhesion, FimH receptor site, type 1 fimbriae

\section{INTRODUCTION}

Bacterial adherence is normally a prerequisite for successful colonization of a specific host tissue. The best characterized group of bacterial adhesins is constituted by fimbriae (for reviews see Klemm, 1994). The most common of the enterobacterial adhesive surface organelles, and one of the best characterized, is type 1 fimbriae. Type 1 fimbriae are found on the majority of Escherichia coli strains and are widespread among other members of the Enterobacteriaceae (Klemm \& Krogfelt, 1994). Interaction between type 1 fimbriae and receptor structures has, in a number of studies, been shown to play a key role in the colonization by $E$. coli of various host tissues (Bloch et al., 1992; Yamamoto et al., 1990). Recently, the adhesion conferred by type 1 fimbriae in an E. coli strain was shown to be linked to urinary tract pathogenesis (Connell et al., 1996). In mouse models, immunization with FimH was shown to prevent urogenital mucosal infection by $E$. coli (Langermann et al.,
1997). A detailed dissection of the adhesive properties of type 1 fimbriae and their role in bacterial receptor recognition would undoubtedly help to reach a clearer understanding of important aspects of bacterial pathogenesis.

A type 1 fimbria is a thin, $7 \mathrm{~nm}$ wide and approximately $1 \mu \mathrm{m}$ long, rod-shaped surface organelle. It is a heteropolymer consisting of four different subunits. Approximately 1000 copies of the major building element, FimA, are polymerized into a right-handed helical structure also containing a small percentage of the minor components FimF, FimG and FimH (Brinton, 1965 ; Klemm \& Christiansen, 1987; Krogfelt \& Klemm, $1988)$. It has been shown that the receptor-recognizing element of type 1 fimbriae is the $30 \mathrm{kDa}$ FimH protein (Krogfelt et al., 1990). The FimH protein is located at the tip and is also interspersed along the fimbrial shaft (Jones et al., 1995; Krogfelt et al., 1990). The FimF and FimG components are probably required for integration 
(a)

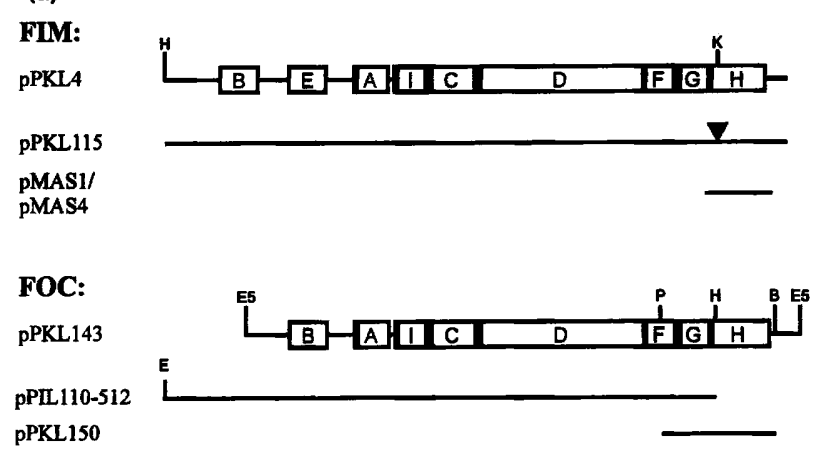

$1 \mathrm{kbp}$

(b)

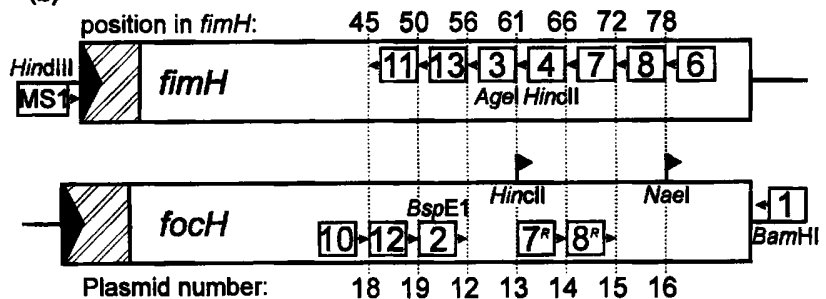

Fig. 1. (a) Overview of the wild-type and engineered versions of the fim and foc gene clusters. Black boxes indicate signalpeptide-encoding sectors. The extent of other recombinant plasmids is indicated by bars. Only DNA inserts are shown. Relevant restriction sites are indicated: B, BamHI; E, EcoRI; E5, EcoRV; $\mathrm{H}$, HindIII; K, Kpnl; P, Pvull. The triangle indicates the position of the translational stop linker insertion. (b) Construction of chimeric fimH-focH genes. Numbers above the fimH gene box indicate the percentage of fimH in the fusions. Positions of primers are indicated by boxed numbers. Restriction sites indicated by flags occur naturally in the focH gene; sites introduced by PCR are written alongside boxed primer numbers.

of the FimH adhesin into the fimbriae (Jones et al., 1995; Klemm \& Christiansen, 1987).

The components of the fimbrial organelle are encoded by the chromosomally located fim gene cluster (Klemm et al., 1985). In addition to the structural components, this $9.5 \mathrm{~kb}$ DNA segment also encodes the fimbrial biosynthesis machinery as well as regulatory elements. Expression is phase-variable with individual cells switching between a fimbriate and a non-fimbriate state. This is due to inversion of a $314 \mathrm{bp}$ DNA segment located immediately upstream of the fim $A$ gene, encoding the major subunit protein (Fig. 1). A promoter residing in this phase switch drives the expression of the fim genes (Olsen \& Klemm, 1994). The elements of the fimbrial organelle are produced as precursors having an $\mathrm{N}$-terminal signal sequence. This feature is subsequently removed during export across the inner membrane. Thus the FimH protein is produced as a precursor of 300 amino acids that is processed into a mature form of 279 amino acids (Hanson et al., 1988; Klemm \& Christiansen, 1987). Further export, from the periplasm and across the outer membrane, is dependent on a fimbriae- specific export and assembly system constituted by the FimC and FimD proteins (Jones et al., 1993; Klemm, 1992; Klemm \& Christiansen, 1990).

By virtue of the FimH adhesin, type 1 fimbriated bacteria confer $\alpha$-D-mannose-sensitive agglutination of a number of eukaryotic cell types displaying this molecular motif, such as certain erythrocytes as well as yeast cells. To investigate more precisely which regions of the FimH adhesin contribute to receptor recognition we have chosen to exploit the compatibility of type 1 fimbriae with another fimbrial system, namely F1C fimbriae. Unlike type 1 fimbriae, F1C fimbriae do not target to Dmannosides; in fact, they do not agglutinate any known red blood cells or yeasts. However, they are known to confer adhesion to an unknown receptor in the collecting ducts and the distal tubules of the human kidney (Virkola et al., 1988), as well as to cultured renal tubulus cells and various cell lines (Kreft et al., 1992; Marre $e t$ al., 1990). The structure of F1C fimbriae closely resembles that of type 1 fimbriae (Riegman et al., 1990; van Die et al., 1984, 1991). They also contain minor components, i.e. the FocF, FocG and FocH proteins. The genetic organization of the two gene clusters encoding the type 1 and F1C fimbriae, viz. the fim and foc genes, is almost identical (Fig. 1). We have previously shown, by reciprocal exchange of minor structural proteins between the type 1 and F1C systems, that the minor components originating from one of these fimbrial systems can be accommodated by the other. Notably, the FimH and FocH proteins, which show $36 \%$ sequence identity, can be readily exchanged between the two systems, resulting in hybrid organelles with receptor specificities defined by the adhesin. The adhesion conferred by FimH integrated in F1C fimbriae precisely mimics that of type 1 fimbriae (Klemm et al., 1994, 1995). The fact that FocH is unable to recognize any of the FimH receptor targets makes it an ideal scaffold into which various parts of the FimH adhesin can be introduced to probe the extent of the receptorbinding domain and associated binding characteristics.

\section{METHODS}

Bacterial strains and growth conditions. In this study, we used a variant of the E. coli K-12 strain HB101 (F' lacI Tn5) (Boyer \& Roulland-Dussoix, 1969) as an intermediate host during plasmid construction work and K-12 strain VL751 [ara $\Delta$ (lac-pro) rpsL thi $\Delta$ fim; Freitag et al., 1985] for phenotype expression. Cells were grown in either glucose minimal medium (Clark \& Maaløe, 1967) containing 0.5\% Casamino acids (Difco) or Luria-Bertani (LB) broth (Sambrook et al., 1989) supplemented with the appropriate antibiotics.

Plasmids. Plasmids used in this study are listed in Table 1. Plasmid pMAS1 contained the fimH gene from plasmid pMAS4 inserted into pUC19. Plasmid pTBK12 was made by introducing an AgeI site at position 536 in the fimH gene by PCR amplification of pMAS1 using primers MS1 and TB3. In parallel, a BspEI site was introduced at position 531 in the focH gene by PCR amplification of pPKL150 (primers TB1 and TB2). The two segments were ligated and the hybrid gene was inserted in pUC19. The gene was subsequently excised 
Table 1. Plasmids

\begin{tabular}{|c|c|c|}
\hline Plasmid & Relevant genotype & Reference/construction \\
\hline pPKL4 & Wild-type fim cluster & Klemm et al. (1985) \\
\hline pPKL115 & $f i m B^{+}-G^{+}$ & Pallesen et al. (1995) \\
\hline pPKL143 & Wild-type foc cluster & Klemm et al. (1994) \\
\hline pPIL110-512 & foc $B^{+}-G^{+}$ & van Die et al. (1984) \\
\hline pPIL110-518 & foc $B^{+}-G^{+}$ & Riegman et al. (1990) \\
\hline pPKL150 & foc $G^{+}$foc $H^{+}$ & Klemm et al. (1994) \\
\hline pMAS1 & $f i m H^{+}$ & This work \\
\hline pMAS4 & $f i m H^{+}$ & Schembri et al. (1996) \\
\hline pTBK16 & fimfocH $\mathrm{H}_{78}$ & First $78 \%$ of $f \mathrm{mH}$ fused to last $22 \%$ of $\mathrm{focH}$ \\
\hline pTBK15 & fimfocH $H_{72}$ & First $72 \%$ of $f i m H$ fused to last $28 \%$ of focH \\
\hline pTBK14 & fimfocH $\mathrm{H}_{66}$ & First $66 \%$ of $f i m H$ fused to last $34 \%$ of focH \\
\hline pTBK13 & fimfocH & First $61 \%$ of $f i m H$ fused to last $39 \%$ of focH \\
\hline pTBK12 & fimfocH $H_{56}$ & First $56 \%$ of $f i m H$ fused to last $44 \%$ of focH \\
\hline pTBK19 & fimfocH $H_{50}$ & First $50 \%$ of $f i m H$ fused to last $50 \%$ of $f o c H$ \\
\hline pTBK18 & fimfocH $\mathrm{H}_{45}$ & First $45 \%$ of $f i m H$ fused to last $55 \%$ of focH \\
\hline
\end{tabular}

and inserted into the unique EcoRV site of pBR322. Plasmid pTBK13 was made by introducing a HincII site at position 573 in the fim $H$ gene by PCR (primers MS1 and TB4), and joining this with the pre-existing $\mathrm{HincII}$ site in the focH gene; this fragment was inserted into EcoRV- and BsmI-digested pBR322. Plasmids pTBK14, pTBK15, pTBK16, TBK18 and TBK19 were made following a similar strategy of PCR amplification of selected segments of the fimH and focH genes, assembly of these in pUC19, and subsequent transfer of the chimeric genes into pBR322 downstream of the tet promoter (Fig. 1). It should be noted that introduction of restriction sites did not alter the amino acid sequence.

DNA techniques. Isolation of plasmid DNA was carried out by the mini-lysate method of Del Sal et al. (1989). Restriction endonucleases were used according to the manufacturer's specifications (New England Biolabs or Pharmacia). PCRs were made as previously described by Stentebjerg-Olesen $\mathrm{et} \mathrm{al}$. (1997). The following primers were used: MS1, 5'-GTGATAAGCTTCACCTATACCTACCTACAGC; TB1, 5'TGTACGGATCCGCCACAACTGCATTCTAG; TB2, $5^{\prime}$ TCCAGTCCGGAACATGCAGGGTCTCATC; TB3, 5'CAGCCACCGGTAGGCACCACCACATC; TB4, 5'-GGCAGGTTAACGGTGACATCACG; TB6, 5'-ACGCCGCCGGCAGGTGAAAACGACG; TB7, 5'-GTTCAGTGCCAATTCCTCTTACTGTACGTTGCGACC; TB8, 5'-GTATTACCTCTCCGGCACACACACAGGAAGTGGTAATACTGTATTC; TB9, 5'-ACAACTATAACAGCGATG; TB10, 5' $^{\prime}$ GCGATTAAAGCTGGCTCATTAGTGGCCACAGTTTATG; TB11, 5'-CATAAACTGTGGCCACTAATGAGCCAGCTTTAATCGC; TB12, 5'-GACCAACAACTATAACAGCGAGAGAAATTTCACCTGGCG; TB13, 5'-CGCCAGGTGGAATTTCTCTCGCTGTTATAGTTGTTGGTC; TB14, 5'-ACTCT'TTCCCCAACAGTTAATGTGGGGCAAAACCTGGTCG; TB15, 5'-CTGGTTTTGCCCCACATTAACTGTTGGGGAAAGAGTGATATTG; TB16, 5'-GTGTTATGTCAGAATGAATATCCGGAAACCATTACAGAC; TB17, 5'-TGTAATGGTTTCCGGATATTCATTCTGACATAACACCAG; TB18, 5'-GCAAGCTTACTGCTACCTATACCTATGCG. The nucleotide sequences of constructs were determined by the dideoxynucleotide chain-termination method (Sanger et al., 1977). Oligonucleotide primers were purchased from Gibco-BRL.
Immunofluorescence microscopy. Surface presentation of FimH-FocH chimeras was assessed by immunofluorescence microscopy employing a monoclonal antibody that recognizes a similar conformational epitope in $\mathrm{FimH}$ and $\mathrm{FocH}$ (Chanteloup et al., 1991). Cell fixation, immunolabelling and microscopy were carried out as previously described by Pallesen et al. (1995).

Electron microscopy. Electron microscopy was carried out essentially as described previously (Klemm et al., 1994; Pallesen et al., 1995). In short, a $10 \mu \mathrm{l}$ aliquot of bacterial suspension was placed on a carbon-coated, glow discharged grid for $30 \mathrm{~s}$. Grids were washed in two drops of PBS $(0.145 \mathrm{M}$ $\mathrm{NaCl} ; 0.15 \mathrm{M}$ sodium phosphate), dehydrated in increasing concentrations of ethanol, blotted dry, and shadowed with tungsten.

Receptor blot. Receptor blots of FimH and FimH-FocH to $\alpha$ D-mannosylated BSA (Sigma) were carried out essentially as previously described by Krogfelt et al. (1990). In short, filter blots were first incubated with $\alpha-D-m a n n o s e-B S A\left(0.5 \mathrm{mg} \mathrm{l}^{-1}\right.$; Sigma) followed by incubation with rabbit anti-BSA serum and finally with peroxidase-conjugated anti-rabbit serum.

Agglutination of red blood cells and yeast cells. The capacity of bacteria to express a D-mannose-binding phenotype was assayed by their ability to agglutinate guinea pig erythrocytes or yeast cells on glass slides. Aliquots of washed bacterial suspensions at $\mathrm{OD}_{550} 0.5$ and $5 \%$ erythrocytes or yeast cells were mixed and the time until agglutination occurred was measured. Furthermore, clones which did not cause any agglutination under these conditions were also tested at $\mathrm{OD}_{550}$ 20 and/or low temperature, but still did not react.

Binding to mannose beads and yeast cells. Binding of bacteria to mannose beads and yeast cells was carried out essentially as described previously (Klemm et al., 1990). In short, bacteria from exponentially growing cultures were harvested, washed three times, and resuspended in PBS. Aliquots of bacteria, with identical $O D_{550}$ values in all cases, and beads (methyl- $\alpha$ D-mannopyranoside-coated beaded agarose; Sigma) or yeast cells (Saccharomyces cerevisiae) were mixed and incubated under gentle agitation for $20 \mathrm{~min}$. Beads or yeast cells were collected by low-spin centrifugation, gently washed and examined microscopically. Binding to beads and yeast cells 
was additionally assayed by incubating aliquots of bacteria with either beads or yeast cells for $2 \mathrm{~min}$, after which bacteria adhering to beads or yeast cells were separated from unbound bacteria by low-speed centrifugation. Unbound bacteria were removed by aspiration, the procedure was repeated twice, and finally attached bacteria were released by addition of methyl $\alpha$ D-mannose. Microscopy revealed that no bacteria remained attached to the beads or yeast cells at this point. The bacteria were then quantified by plating out, the experiments were repeated four times.

Adhesion inhibition assay. Inhibitor titration, with methyl $\alpha$ D-mannose, of bacterial binding to mannan bound to 96-well plates was carried out essentially as previously described by Sokurenko et al. (1997). In short, wells were coated with mannan (Sigma) at a concentration of $10 \mu \mathrm{g} \mathrm{ml}^{-1}$, washed three times with PBS and quenched with $0.2 \%$ BSA in PBS. Bacterial suspensions containing identical cell numbers were mixed with increasing concentrations of methyl $\alpha$-D-mannose, added to the wells and incubated for $40 \mathrm{~min}$ at $37^{\circ} \mathrm{C}$. The wells were then washed with PBS, and $160 \mu \mathrm{l} \mathrm{LB}$ containing $100 \mathrm{mM}$ methyl $\alpha$-D-mannose was added to each well and incubated for $5 \mathrm{~h}$ at $37^{\circ} \mathrm{C}$. The number of bound bacteria was determined by measuring $\mathrm{OD}_{600}$ values with a microtitre plate reader.

\section{RESULTS}

\section{Construction of genes encoding hybrid FimH-FocH proteins}

A series of chimeric genes was created which harboured sectors of the fim $\mathrm{H}$ gene encoding 45, 50, 56, 61, 66, 72 or $78 \%$ of the $\mathrm{N}$-terminal part of the FimH protein. These sectors were fused to segments of the focH gene encoding the complementary C-terminal sectors of the FocH protein. The result was a nested series of genes encoding FimH-FocH fusions containing increasingly larger segments of the $\mathrm{N}$-terminal part of the FimH protein, i.e. between 125 and 220 amino acid residues, fused to correspondingly smaller segments of the Cterminal part of the $\mathrm{FocH}$ protein (Table 1; Fig. 1). DNA sequence analysis established that the chimeras authentically mimicked the expected sectors of the parent genes and that the reading frame was conserved in the fusion points in all cases.

\section{Agglutination conferred by FimH-FocH hybrids}

To investigate the binding potential of the FimH-FocH hybrids, we initially performed 'classic' agglutination assays. Auxiliary plasmids, encoding either all the fim genes except fim $H$ or all the foc genes except focH, were used to complement plasmids encoding the FimH-FocH chimeras. The resulting hosts therefore display the chimeras as either constituents of type 1 or F1C fimbriae, respectively. Hybrid FimH-FocH containing 72 or $78 \%$ of the $\mathrm{N}$-terminal part of FimH conferred agglutination times that were only slightly slower than the wild-type FimH displayed by either of the parent fimbrial systems (Table 2). Furthermore, the titres were basically unaffected by the display system, i.e. either type 1 or F1C fimbriae. However, substitution of larger sectors between FimH and FocH, i.e. the $45-66 \%$ fusions, was unable to induce agglutination of erythrocytes and yeast cells, which provide a rich source of mannan (Table 2 ).

\section{Surface presentation of the adhesins}

Surface presentation of the FimH-FocH hybrids was assessed by immunofluorescence microscopy employing a monoclonal antibody which recognizes an identical conformational epitope in both FimH and FocH. In all cases, similar signals that differed significantly from the negative controls were observed. In addition, fimbrial morphology and number were assessed by electron microscopy. Two representative recombinant strains were investigated, i.e. cells containing plasmids pPKL115 plus pTBK14 expressing the FimH-FocH hybrid adhesin and cells containing plasmids pPKL115 plus pTBK15 expressing the FimH-FocH ${ }_{72}$ hybrid adhesin, and compared to the wild-type control (Fig. 2). In both cases, fimbriated cells were seen where the morphology and number of the fimbriae were similar to the wild-type.

\section{D-Mannose recognition by FimH-FocH hybrids}

The binding capacity of the chimeras was also assessed by a different parameter, namely the ability to bind to $D$ mannosylated BSA. In this assay, it was found that FimH-FocH hybrids consisting of $56 \%$ or more of the $\mathrm{N}$-terminal region of FimH fused to the complementary C-terminal sectors of $\mathrm{FocH}$, readily bound to mannose-BSA. By fivefold overloading of the gel, a weak signal was observed with the $50 \%$ fusion, whereas the $45 \%$ fusion did not react under these conditions (Fig. 3). Addition of methyl $\alpha$-D-mannose to the assay completely abolished binding to mannose-BSA.

\section{D-Mannose recognition is distinct from the ability to cause agglutination}

At this point it was apparent that the ability of a FimH-FocH hybrid to recognize D-mannose was not concomitant with the ability to cause agglutination of erythrocytes or yeast cells. All hybrids containing $56 \%$ or more of the FimH N-terminus clearly recognized mannose-BSA but only hybrids containing $72 \%$ or more were able to induce agglutination (Table 3 ). To investigate this aspect further, we looked at the ability of the clones to bind to methyl- $\alpha$-D-mannopyranosidecoated beaded agarose. It was found that exactly the same hybrids, i.e. the $56,61,66,72$ and $78 \%$ fusions, that readily bound to mannose-BSA in the receptor blot assay also conferred binding of bacteria to the beads (Fig. 4). The 45 and $50 \%$ fusions did not differ from the negative control. Quantification of the percentage of bacteria adhering to the monosaccharide mannose target on the beads revealed only minor differences among the recombinant strains. The exceptions were the negative control, expressing $\mathrm{FocH}$, and to a degree the wild-type control (Fig. 4).

Additionally, we investigated the ability of the hybrids to bind to yeast cells, since the receptor target, mannan, 
Table 2. Adhesive phenotypes conferred by FimH-FocH hybrids expressed in both type 1 and $F 1 C$ backgrounds assayed by the ability to agglutinate guinea pig erythrocytes of $E$. coli VL751 ( $\Delta$ fim) derivatives

Agglutination of guinea pig erythrocytes or yeast cells is indicated in seconds before reaction occurred. The mean values of three measurements are given.

\begin{tabular}{|c|c|c|c|}
\hline Plasmid(s) & $\begin{array}{l}\text { Relevant } \\
\text { genotype }\end{array}$ & Haemagglutination & $\begin{array}{c}\text { Yeast } \\
\text { agglutination }\end{array}$ \\
\hline pMAS4 & $f i m H^{+}$ & & \\
\hline +pPKL115 & $\mathrm{fim} B^{+}-G^{+}$ & 12 & 9 \\
\hline pMAS4 & $\mathrm{fimH}^{+}$ & & \\
\hline +pPIL110-512 & foc $B^{+}-G^{+}$ & 10 & 8 \\
\hline pTBK16 & fimfocH $H_{38}$ & & \\
\hline+ pPKL115 & $f i m B^{+}-G^{+}$ & 19 & 11 \\
\hline pTBK16 & fimfocH $_{78}$ & & \\
\hline +pPIL110-512 & foc $B^{+}-G^{+}$ & 24 & 13 \\
\hline pTBK15 & fimfoc $_{72}$ & & \\
\hline +pPKL115 & fimB $B^{+}-G^{+}$ & 20 & 12 \\
\hline pTBK15 & fimfocH $_{72}$ & & \\
\hline +pPIL110-512 & foc $B^{+}-G^{+}$ & 26 & 13 \\
\hline pTBK14 & fimfoc $H_{66}$ & & \\
\hline+ pPKL115 & $f i m B^{+}-G^{+}$ & $>600$ & $>600$ \\
\hline pTBK14 & fimfocH $H_{66}$ & & \\
\hline +pPIL110-512 & foc $B^{+}-G^{+}$ & $>600$ & $>600$ \\
\hline pTBK13 & fimfocH $_{61}$ & & \\
\hline+ pPKL115 & fim $B^{+}-G^{+}$ & $>600$ & $>600$ \\
\hline pTBK13 & fimfocH $_{61}$ & & \\
\hline+ pPIL110-512 & foc $B^{+}-G^{+}$ & $>600$ & $>600$ \\
\hline pTBK12 & fimfocH $_{56}$ & & \\
\hline+ pPKL115 & fimB $B^{+}-G^{+}$ & $>600$ & $>600$ \\
\hline pTBK12 & fimfocH $H_{56}$ & & \\
\hline +pPIL110-512 & foc $B^{+}-G^{+}$ & $>600$ & $>600$ \\
\hline рTBK19 & fimfoc $\mathrm{H}_{50}$ & & \\
\hline+ pPKL115 & $f\left(m B^{+}-G^{+}\right.$ & $>600$ & $>600$ \\
\hline pTBK19 & fimfoc $_{50}$ & & \\
\hline+ pPIL110-512 & foc $B^{+}-G^{+}$ & $>600$ & $>600$ \\
\hline pTBK18 & fimfoch $H_{45}$ & & \\
\hline+ pPKL115 & fimB $B^{+}-G^{+}$ & $>600$ & $>600$ \\
\hline pTBK18 & fimfoc $_{45}$ & & \\
\hline +pPIL110-512 & $f \circ B^{+}-G^{+}$ & $>600$ & $>600$ \\
\hline pPKL143 & Wild-type foc cluster & $>600$ & $>600$ \\
\hline pPKL115 & $f i m B^{+}-G^{+}$ & $>600$ & $>600$ \\
\hline pPIL110-512 & $f \circ c B^{+}-G^{+}$ & $>600$ & $>600$ \\
\hline
\end{tabular}

is different from the $\alpha$-D-mannose or the methyl $\alpha$-Dmannopyranoside targets on mannose-BSA and mannose-beads, respectively. Microscopy of yeast cells incubated with the individual clones revealed that the 72 and $78 \%$ fusions, which were able to induce agglutination, also attached to the yeast cells (Fig. 4). Surprisingly, the non-agglutinating clones, expressing FimH-FocH ${ }_{56}$ through FimH-FocH ${ }_{66}$, were also seen to attach to yeast cells (Fig. 4). Enumeration of attached bacteria, however, revealed two distinct groups. In one group conferring agglutination, between 65 and $85 \%$ of the added bacteria bound to yeast, whereas in the nonagglutinating group only between 30 and $45 \%$ of the bacteria adhered to the yeast cells under the assay conditions (Fig. 4). Judging from the above, a Dmannose-recognizing phenotype seemed to be distinct from and superimposed on the ability to confer agglutination. It should also be noted that the binding phenotype of the hybrids was independent of whether type 1 or F1C fimbriae were used as display organelles.

\section{Inhibition tests}

To probe the binding of the hybrids from a different angle, we also looked at the inhibition profiles, i.e. the ability of recombinant cells to bind to mannan in the 

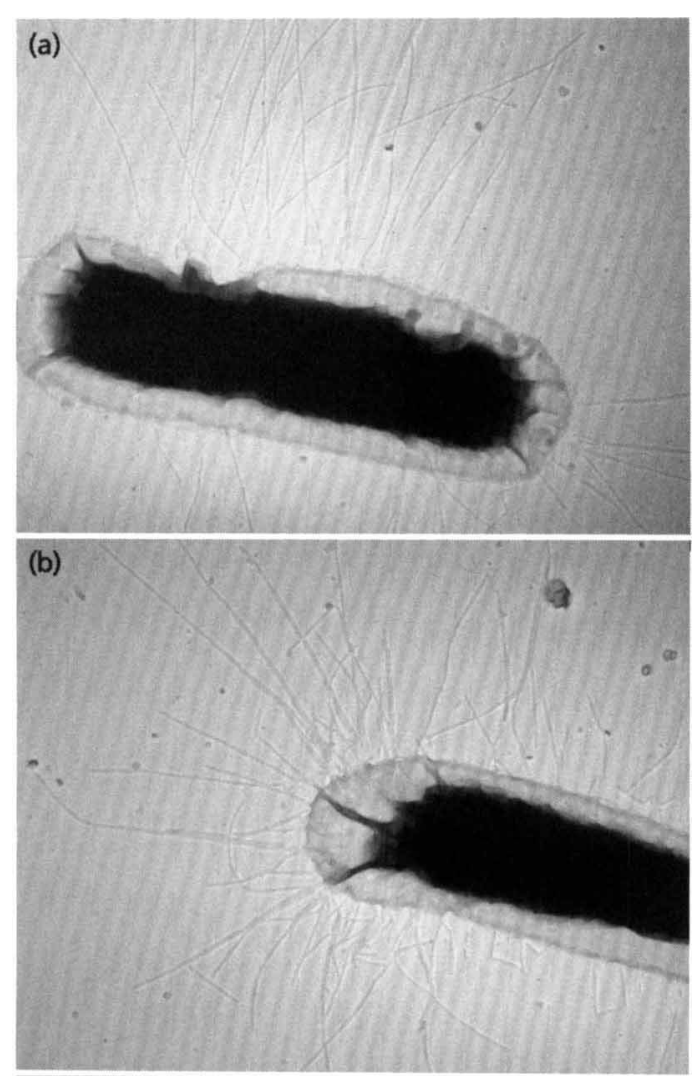

(c)

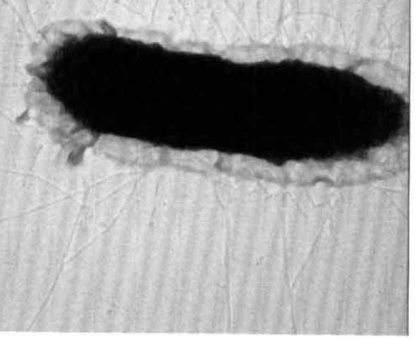

Fig. 2. Electron micrographs showing fimbriation of $E$. coli strain VL751 cells containing recombinant plasmids which in addition to a fim gene cluster with a stop-linker in the fim $H$ gene encode the following adhesins: (a) $\mathrm{FimH}_{-} \mathrm{FocH}_{72}$ (pTBK15 + pPKL115); (b) wild-type FimH (pMAS4 + pPKL115); (c)

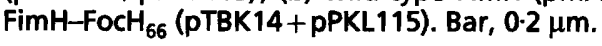

presence of increasing concentrations of methyl $\alpha-\mathrm{D}-$ mannoside (Fig. 5). The binding of all strains to mannan could be inhibited by methyl $\alpha$-D-mannoside; however, the concentrations of methyl $\alpha$-D-mannoside required to inhibit adhesion by $50 \%$, viz. the $\mathrm{IC}_{50}$, differed significantly among the strains. Two groups were apparent, with values differing by about one order of magnitude. One group which conferred agglutination and D-mannose binding, i.e. cells expressing FimH, FimH-FocH and $\mathrm{FimH}-\mathrm{FocH}_{72}$, had $\mathrm{IC}_{50}$ values of $1.60,4.7$ and
$0.80 \mathrm{mM}$, respectively, whereas hybrids unable to cause agglutination but which still bound to mannose, i.e. FimH-FocH ${ }_{66}$, FimH-FocH ${ }_{61}$ and FimH-FocH 5 , had $\mathrm{IC}_{50}$ values of 0.08 (extrapolated), 0.10 and $0.20 \mathrm{mM}$, respectively.

\section{DISCUSSION}

One of the decisive factors determining the range of host organisms, tissues and cell types that bacteria are able to colonize is their ability to recognize and bind to specific receptor molecules on host cell surfaces. In the case of fimbriae-mediated adhesion, this target tropism is determined by the molecular architecture of the binding domain of the fimbrial adhesin. The archetypical fimbrial adhesin is undoubtedly the FimH adhesin, a constituent of the (among enterobacteria) ubiquitously present type 1 fimbriae. The type 1 fimbriae with the associated adhesive phenotype was one of the first bacterial adhesins to be described and defined by virtue of its haemagglutinating activity which could be inhibited by the simple monosaccharide $\alpha$-D-mannose (Duguid et al., 1955; Duguid \& Gillies, 1957). Since then, a number of powerful tools from the domains of biochemistry, genetics and molecular biology have disclosed many interesting features of this adhesin. In the present study, we have used the compatibility of FimH and FocH with either of the two parental organelle systems to display chimeric versions of the two proteins on the bacterial surface to study the receptor-binding domain of FimH. The inability of FocH to recognize any of the receptor targets of FimH makes it an ideal scaffold onto which sectors of FimH can be grafted. Proteins or protein domains with identity scores above $20 \%$ are generally considered to have similar three-dimensional structures (Chotia \& Lesk, 1986; Sander \& Schneider, 1991). The primary structures of the FimH and FocH proteins exhibit an overall $36 \%$ identity indicative of similar folding. However, it is noteworthy that the homology between the proteins is not evenly distributed, and the homologies of the $\mathrm{N}$-terminal and C-terminal halves of the proteins are below or above this mean, respectively.

The adhesive capacity of the FimH-FocH fusions was tested against two classes of receptor molecules containing either a single $\alpha$-D-mannose or methyl $\alpha$-Dmannose residue coupled to BSA or agarose beads, or complex $\alpha$-D-mannose-containing polysaccharides as present on yeast cells (a rich source of mannan), mannan and guinea pig red blood cells. Hybrids which contained $72 \%$ or more of the N-terminal part of FimH fused to the corresponding C-terminal parts of $\mathrm{FocH}$ were shown to confer binding characteristics resembling those of the wild-type FimH adhesin, i.e. agglutination of red blood cells and yeast cells (Table 2). Cells expressing such hybrids also bound in high numbers to yeast cells and methyl- $\alpha$-D-mannopyranoside-conjugated beaded agarose (Fig. 4); the hybrid proteins were shown to bind to mannose-BSA (Fig. 3). Surprisingly, chimeras harbouring less of the $\mathrm{N}$-terminal part of FimH, viz. from 


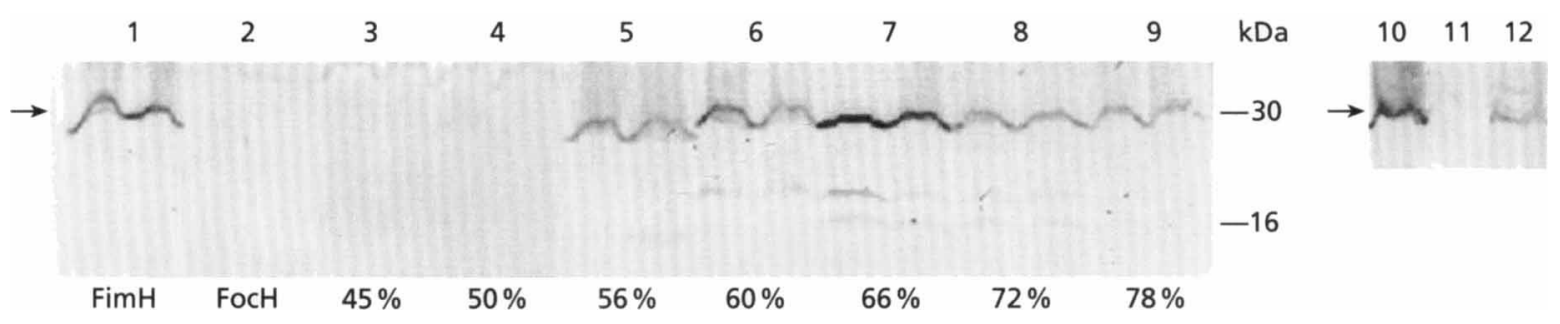

Fig. 3. Analysis of binding to $\alpha$-D-mannose-BSA by FimH-FocH hybrids. Equivalent samples were subjected to SDS-PAGE and transferred to a PVDF filter. Bound $\alpha$-D-mannose-BSA was detected by reaction with anti-BSA rabbit serum followed by peroxidase-conjugated anti-rabbit serum. Lanes are shown in doublets with the adhesin expressed by type 1 or F1C fimbriae, respectively. The position of the FimH-FocH hybrids is indicated by an arrow. Lanes: 1, wild-type FimH; 2, wild-

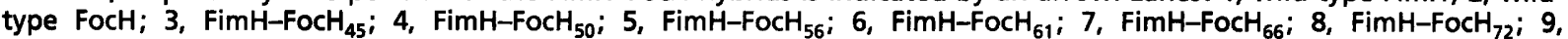
$\mathrm{FimH}-\mathrm{FoCH}_{78}$. The insert on the right-hand side represents a fivefold overloading of the gel with equivalent samples of wild-type FimH (lane 10), wild-type FocH (lane 11) and FimH-FocH ${ }_{50}$ (lane 12).

Table 3. Summary of phenotypes conferred by FimH-FocH hybrids

The extent of FimH in the hybrids is indicated. In all cases, a helper plasmid encoding all fim genes except fimH was also present in the host strain (E. coli VL751).

\begin{tabular}{|c|c|c|c|c|c|}
\hline Hybrid & $\begin{array}{c}\text { Residues of mature } \\
\text { FimH }\end{array}$ & $\begin{array}{c}\text { Mannose-BSA } \\
\text { binding }\end{array}$ & $\begin{array}{l}\text { Mannose-bead } \\
\text { binding }\end{array}$ & $\begin{array}{c}\text { Yeast cell } \\
\text { binding }\end{array}$ & Agglutination ${ }^{*}$ \\
\hline FimH & $1-279$ & + & + & + & + \\
\hline $\mathrm{FimH}_{-} \mathrm{FocH}_{78}$ & $1-217$ & + & + & + & + \\
\hline $\mathrm{FimH}_{-} \mathrm{FocH}_{72}$ & $1-201$ & + & + & + & + \\
\hline FimH-FocH 6 & $1-184$ & + & + & + & - \\
\hline FimH-FocH & $1-170$ & + & + & + & - \\
\hline $\mathrm{FimH}-\mathrm{FocH}_{56}$ & $1-158$ & + & + & + & - \\
\hline $\mathrm{FimH}-\mathrm{FocH}_{50}$ & $1-139$ & $(+)$ & - & - & - \\
\hline FimH-FocH 45 & $1-125$ & - & - & - & - \\
\hline FocH & 0 & - & - & - & - \\
\hline
\end{tabular}

* Agglutination of guinea pig erythrocytes or yeast cells.

56 through $66 \%$, were unable to confer agglutination but were nevertheless able to bind to D-mannose-BSA, methyl- $\alpha$-D-mannopyranoside-conjugated beaded agarose and yeast cells (Table 2; Figs 3 and 4). It should be mentioned that such clones were agglutination-negative, even when the assays were carried out with concentrated bacterial suspensions and/or at low temperature. This suggests that D-mannose binding and agglutination actually can be distinct phenotypes and that the two characteristics can be related to physically distinct regions in FimH. Finally, the $50 \%$ fusion did bind weakly to D-mannose-BSA, but was negative in all other binding assays. The $45 \%$ fusion tested negatively by all criteria we used to monitor binding with, indicative of the extent of the D-mannose-binding domain of FimH. Taken together, our results from the chimeric FimH-FocH approach suggest that the minimal information for D-mannose recognition in FimH is located within the first $50 \%$ of FimH. Furthermore, this 'core domain' can be complemented with steric information residing in the remaining part of FimH. Notably, the 185-201 segment apparently contains information that upgrades the D-mannose-binding core domain to additionally confer agglutination, reflected in the different binding phenotypes endowed by $\mathrm{FimH}-\mathrm{FocH}_{66}$ and FimH-FocH ${ }_{72}$.

Two other sets of observations indicate that the agglutinating- and mannose-binding-only phenotypes are probably a reflection of higher avidity for the receptor target in the first phenotype. Mannan is a branching polymer consisting of several hundred mannose residues (Ballou, 1976). When presented with such a target, fusions having $72 \%$ or more of the N-terminal sector of FimH bound in significantly higher numbers to yeast cells and had higher $\mathrm{IC}_{50}$ values than hybrids that only conferred binding to monosaccharide mannose targets (Figs 4 and 5). Utilizing a large spectrum of saccharides, Sharon and coworkers found that the binding domain of FimH is probably an elongated pocket that recognizes a trisaccharide motif (Firon et al., 1987; Sharon, 1987). Increased affinity for oligosaccharides by many lectins is derived from extended binding sites in which more than just terminal sugar residues of an oligosaccharide make 

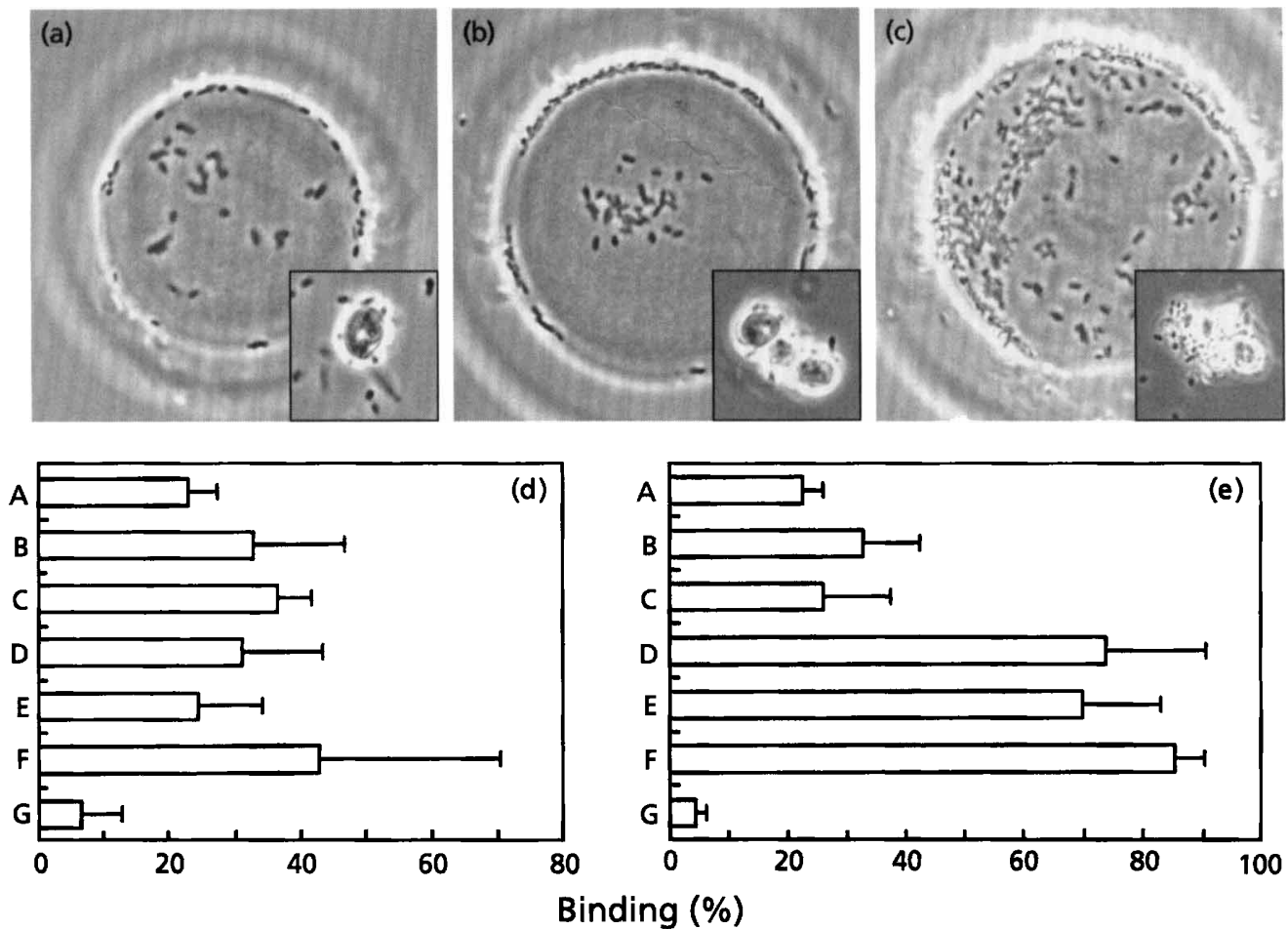

Fig. 4. Bacterial attachment to monosaccharide (methyl- $\alpha$-D-mannopyranoside-coated beads) and polysaccharide (yeast cells) targets. (a-c) Micrographs of $E$. coli strain VL751 expressing FimH-FocH 61 (pTBK13+pPKL115), FimH-FocH (pTBK16 + pPKL115) and FimH (pMAS4 + pPKL115), respectively, adhering to beads and yeast cells (inserts). The block diagrams in (d) and (e) show binding of recombinant bacteria to mannose-beads and yeast, respectively. The adhesin

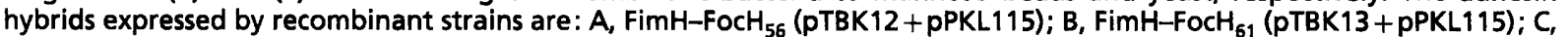
FimH-FocH $_{66}$ (pTBK14+pPKL115); D, FimH-FocH $_{72}$ (pTBK15+pPKL115); E, FimH-FocH 78 (pTBK16+pPKL115); F, FimH (pMAS4 + pPKL115); G, FocH (pPKL143). The results shown are the means of four experiments \pm SD. For experimental details see Methods.

contact (Weis \& Drickamer, 1996). In the light of this, it is tempting to speculate that such an elongated binding site in FimH, capable of accommodating an extended oligosaccharide motif, is gradually affected in our FimH-FocH fusions when we decrease the FimH portion, resulting in different binding characteristics. We also constructed reverse fusions, i.e. focH-fimH hybrids encoding N-terminal sectors of $\mathrm{FocH}$ fused to complementary regions of FimH. However, all efforts to detect the corresponding gene products or related phenotypes were unsuccessful.

Previous comparisons of FimH variants showed that minor differences in the amino acid sequence resulted in different binding characteristics of the adhesin (Sokurenko et al., 1994, 1995, 1997). Such differences were located between residues 32 and 166 (Fig. 6). Further information on the structure-function architecture of the FimH adhesin was provided by linker insertion analysis of the fim $\mathrm{H}$ gene. A systematic change of codons in the wild-type sequence in a number of positions, spanning the entire sequence, resulted in a series of FimH variants with a wide range of adhesive phenotypes. Notably, changes in positions 56 and $136 / 137$ abolished receptor recognition, whereas ma- nipulation of positions 8, 225 and 259 did not (Schembri et al., 1996). In addition, a 16-18 kDa truncate of FimH, having the same $\mathrm{N}$-terminal sequence as the mature FimH protein, was reported to confer binding to Dmannose since it was isolated from D-mannose beads (Jones et al., 1995). Comparable-sized truncates were also observed in this study indicative of similar processing of the chimeric adhesins (Fig. 3). Very recently it was reported (Thankavel et al., 1997) that a MalE fusion containing residues 1-100 of FimH (actually residues $3-102$, according to the primer sequences) recognized $\alpha$ D-mannose-containing targets. A weak signal, about 25 fold weaker than the other signals, could indeed be seen with FimH-FocH $\mathrm{H}_{50}$ in the mannose-BSA receptor blot (Fig. 3). Overloading of the gel confirmed this observation. This would suggest that some modicum of $\mathrm{D}$ mannose-binding activity can be ascribed to the first $50 \%$ of FimH.

Although a peptide segment encompassing the $\mathrm{N}$ terminal $50 \%$ of FimH seems to confer binding to Dmannose targets, sectors outside this segment heavily influence the receptor binding. These are perhaps involved in accommodation of more extensive targets than just a single monosaccharide motif. Previous 


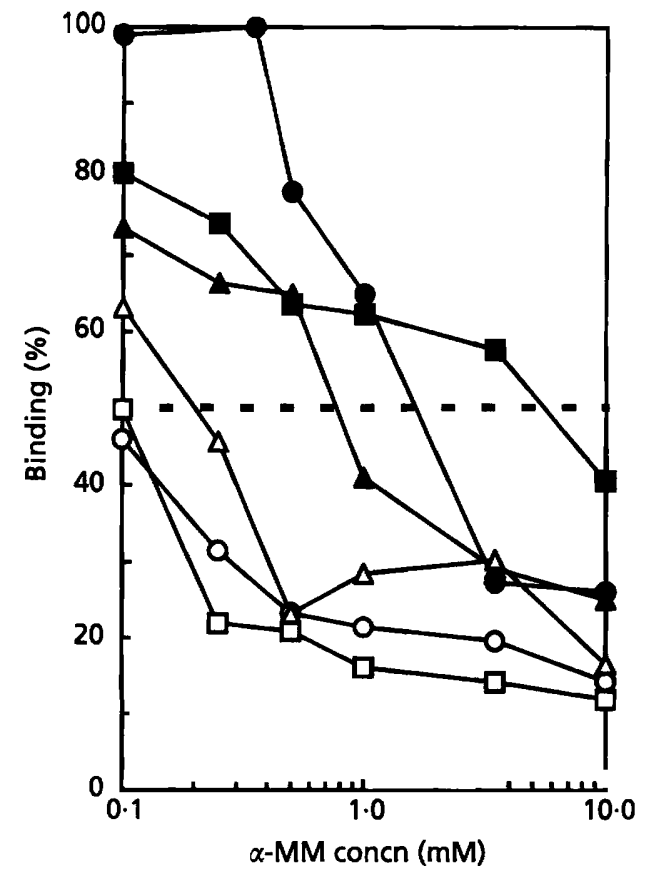

Fig. 5. Inhibition of binding of strains expressing hybrid adhesins to mannan by methyl $\alpha-D-$ mannopyranoside $(\alpha-M M)$. Data were converted to percentage of bound cells by correlating with the number of cells bound in the absence of inhibitor. The dashed line indicates $I_{50}$. FimH; FimH-FoCH 78 ; $\Delta$, FimH-FocH ${ }_{72} ;$ O, FimH-FocH ${ }_{66} ; \square$, FimH-FocH ${ }_{61} ; \triangle$, FimH-FocH ${ }_{56}$. Data from a single experiment are shown, but the experiment was repeated several times and the results were essentially the same.

findings also confirm this (Sokurenko et al., 1994, 1995). The minimal sector of FimH that was observed to confer binding to a D-mannose monosaccharide target with the FimH-FocH technique was contained within the $\mathrm{N}$ terminal 140 residues of FimH. The importance of this region can also be seen from the fact that abolition of receptor recognition was previously reported by sitedirected mutagenesis causing alteration, but not deletion, of the amino acids in positions 136 and 137 (Schembri et al., 1996). Also, in this study we find that a segment straddling position 200 seems to be involved in the modulation of receptor binding of FimH since replacement of this segment with the corresponding FocH segment causes abolition of agglutination but not of D-mannose recognition. It therefore seems that although part of the FimH receptor recognition domain appears to be restricted to the first half of the molecule, other sectors of the protein must significantly contribute to the receptor recognition. The modulating effect of such regions on FimH receptor recognition might only be observable in the context of intact fimbrial organelles as reported here. The present approach, using fusion constructs of two homologous adhesins to dissect receptor-binding sites within one of them, is novel and should have a high potential for the study of structure-function relationships of bacterial adhesins.

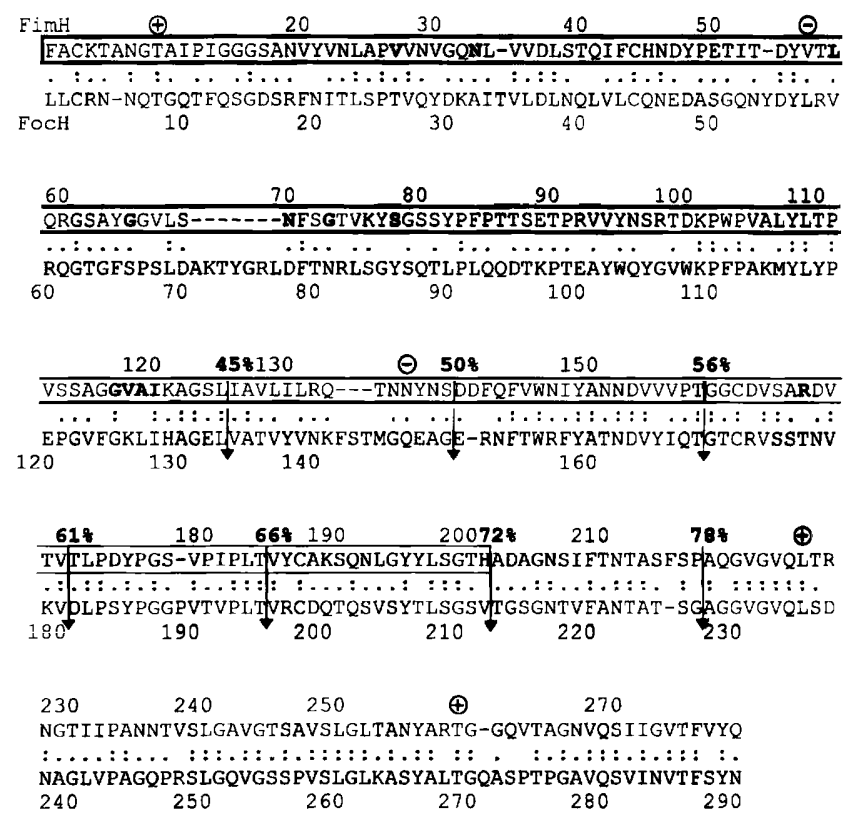

Fig. 6. Comparison of the amino acid sequences of the mature FimH and FocH proteins. Gaps are included to obtain maximum fit. FimH-FocH hybrids are indicated by arrows and percentage of FimH in the chimeric protein. Identical amino acids are indicated by colons and functionally similar amino acids with stops. Positions in which amino acid changes alter binding phenotype (Sokurenko et al., 1994, 1995) are indicated in bold. Binding characteristics of FimH variants created by linkerinsertion or site-directed mutagenesis (Schembri et al., 1996) are indicated by circled ' + ' and ' - '. The minimum segments of FimH which in the context of FimH-FocH hybrids were required to confer efficient D-mannose binding and additionally agglutination are indicated by boxing with thick or thin lines, respectively.

\section{ACKNOWLEDGEMENTS}

This work was supported by the Danish Technical and Natural Sciences Research Councils. We greatly appreciate the electron microscopy work expertly carried out by Gunna Christiansen. We also wish to thank David Hasty, Hugh Connell, Hakon Leffler and Mark Schembri for constructive criticism and comments.

\section{REFERENCES}

Ballou, C. (1976). Structure and biosynthesis of mannan component of the yeast cell envelope. Adv Microb Physiol 14, 93-158. Bloch, C. A., Stocker, B. A. D. \& Orndorf, P. E. (1992). A key role for type 1 pili in enterobacterial communicability. Mol Microbiol 6, 697-701.

Boyer, H. W. \& Roulland-Dussoix, D. (1969). A complementary analysis of the restriction and modification of DNA in Escherichia coli. J Mol Biol 41, 459-472.

Brinton, C. C., Jr (1965). The structure, function, synthesis and genetic control of bacterial pili and a molecular model for DNA and RNA transport in Gram negative bacteria. Trans $N$ Y Acad Sci 27, 1003-1054.

Chanteloup, N. K., Dho-Moulin, M., Esnault, E., Brée, A. \& Lafont, J.-P. (1991). Serological conservation and location of the adhesin 
of avian Escherichia coli type 1 fimbriae. Microb Pathog 10, 271-280.

Chotia, C. \& Lesk, M. (1986). The relation between the divergence of sequence and structure in proteins. EMBO J 5, 823-826.

Clark, D. J. \& Maaløe, O. (1967). DNA replication and the division cycle in Escherichia coli. J Mol Biol 23, 99-112.

Connell, H., Agace, W., Klemm, P., Schembri, M., Mărild, S. \& Svanborg, C. (1996). Type 1 fimbrial expression enhances Escherichia coli virulence for the urinary tract. Proc Natl Acad Sci USA 93, 9827-9832.

Del Sal, G., Manfioletti, G. \& Schneider, C. (1989). The CTABDNA precipitation method: a common mini-scale preparation of template DNA from phagemids, phages or plasmids suitable for sequencing. BioTechniques 7, 514-519.

van Die, I., Van Geffen, B., Hoekstra, W. \& Bergmans, H. (1984). Type 1C fimbriae of an uropathogenic Escherichia coli strain: cloning and characterization of the genes involved in the expression of the F1C antigen and nucleotide sequence of the subunit gene. Gene 34, 187-196.

van Die, I., Kramer, C., Hacker, J., Bergmans, H., Jongen, W. \& Hoekstra, W. (1991). Nucleotide sequence of the genes coding for the minor fimbrial subunits of the F1C fimbriae of Escherichia coli. Res Microbiol 142, 653-658.

Duguid, J.P. \& Gillies, R. R. (1957). Fimbriae and adhesive properties in dysentery bacilli. J Pathol Bacteriol 74, 397-411.

Duguid, J. P., Smith, I. W., Dempster, G. \& Edmonds, P. N. (1955). Non-flagellar filamentous appendages (fimbriae) and haemagglutinating activity in Bacterium coli. J Pathol Bacteriol 70, 335-348.

Firon, N., Ashkenazi, S., Mirelman, D., Ofek, I. \& Sharon, N. (1987). Aromatic alpha-glycosides of mannose are powerful inhibitors of the adherence of type 1 fimbriated Escherichia coli to yeast and intestinal epithelial cells. Infect Immun 55, 472-476.

Freitag, C. S., Abraham, J. M., Clements, J. R. \& Eisenstein, B. I. (1985). Genetic analysis of the phase variation control of expression of type 1 fimbriae in Escherichia coli. J Bacteriol 162, 668-675.

Hanson, M. S., Hempel, J. \& Brinton, C. C., Jr (1988). Purification of the Escherichia coli type 1 minor pilin proteins and partial characterization of the adhesin protein. $J$ Bacteriol 170, 3350-3358.

Jones, C. H., Pinkner, J. S., Nicholes, A. V., Slonin, L. N., Abraham, S. N. \& Hultgren, S. J. (1993). FimC is a periplasmic PapD-like chaperone that directs assembly of type 1 pili in bacteria. Proc Natl Acad Sci USA 90, 8397-8401.

Jones, C. H., Pinkner, J. S., Roth, R., Heuser, J., Nicholes, A. V., Abraham, S. \& Hultgren, S. J. (1995). FimH adhesin of type 1 pili is assembled into a fibrillar tip structure in the Enterobacteriaceae. Proc Natl Acad Sci USA 92, 2081-2085.

Klemm, P. (1992). FimC, a chaperone-like periplasmic protein of Escherichia coli involved in biogenesis of type 1 fimbriae. Res Microbiol 143, 831-838.

Klemm, P. (editor) (1994). Fimbriae, Adbesion, Genetics, Biogenesis and Vaccines. Boca Raton, FL: CRC Press.

Klemm, P. \& Christiansen, G. (1987). Three fim genes required for the regulation of length and mediation of adhesion of Escherichia coli type 1 fimbriae. Mol Gen Genet 208, 439-445.

Klemm, P. \& Christiansen, G. (1990). The fimD gene required for cell surface localization of Escherichia coli type 1 fimbriae. Mol Gen Genet 220, 334-338.

Klemm, P. \& Krogfelt, K. A. (1994). Type 1 fimbriae of Escherichia coli. In Fimbriae, Adbesion, Genetics, Biogenesis and Vaccines, pp. 9-26. Edited by P. Klemm. Boca Raton, FL: CRC Press.

Klemm, P., Jørgensen, B. J., van Die, I., de Ree, H. \& Bergmans, H. (1985). The fim genes responsible for synthesis of type 1 fimbriae in Escherichia coli. Mol Gen Genet 199, 410-414.

Klemm, P., Krogfelt, K. A., Hedegaard, L \& Christiansen, G. (1990). The major subunit of Escherichia coli type 1 fimbriae is not required for D-mannose specific adhesion. Mol Microbiol 4, 553-559.

Klemm, P., Christiansen, G., Kreft, B., Marre, R. \& Bergmans, H. (1994). Reciprocal exchange of minor components of type 1 and F1C fimbriae results in organelles with changed receptor specificities. J Bacteriol 176, 2227-2234.

Klemm, P., Jorgensen, B. J., Kreft, B. \& Christiansen, G. (1995). The export systems of type 1 and $\mathrm{F} 1 \mathrm{C}$ fimbriae are interchangeable but work in parental pairs. J Bacteriol 177, 621-627.

Kreft, B., Carstensen, O., Straube, E., Bohnet, S., Hacker, J. \& Marre, R. (1992). Adherence to and cytotoxicity of Escherichia coli for eucaryotic cell lines quantified by MTT (3-[4,5-dimethylthiazol-2-yl]-2,5-diphenyltetrazolium bromide). Zentbl Bakteriol Parasitenkd Infektkrankb Hyg 276, 231-242.

Krogfelt, K. A. \& Klemm, P. (1988). Investigation of minor components of Escherichia coli type 1 fimbriae: protein chemical and immunological aspects. Microb Pathog 4, 231-238.

Krogfelt, K. A., Bergmans, H. \& Klemm, P. (1990). Direct evidence that the FimH protein is the mannose specific adhesin of Escherichia coli type 1 fimbriae. Infect Immun 58, 1995-1998.

Langermann, S., Palaszynsky, S., Barnhart, M. \& 8 other authors (1997). Prevention of mucosal Escherichia coli infection by FimHadhesin-based systemic vaccination. Science 276, 607-611.

Marre, R., Kreft, B. \& Hacker, J. (1990). Genetically engineered S and F1C fimbriae differ in their contribution to adherence of Escherichia coli to cultured renal tubulus cells. Infect Immun 58, 3434-3437.

Olsen, P. B. \& Klemm, P. (1994). Localization of promoters in the fim gene cluster and the effect of H-NS on the transcription of fimB and fimE. FEMS Microbiol Lett 116, 95-100.

Pallesen, L., Poulsen, L. K., Christiansen, G. \& Klemm, P. (1995). Chimeric FimH adhesin of type 1 fimbriae: a bacterial display system for heterologous sequences. Microbiology 141, 2839-2848.

Riegman, N., Kusters, R., van Veggel, H., Bergmans, H., van Bergen en Henegouwen, P., Hacker, J. \& van Die, I. (1990). F1C fimbriae of a uropathogenic Escherichia coli: genetic and functional organization of the foc gene cluster and identification of minor components. J Bacteriol 172, 1114-1120.

Sambrook, J., Fritsch, E. F. \& Maniatis, T. (1989). Molecular Cloning: a Laboratory Manual, 2nd edn. Cold Spring Harbor, NY: Cold Spring Harbor Laboratory.

Sander, C. \& Schneider, R. (1991). Database of homology-derived protein structures and the structural meaning of sequence alignment. Proteins 9, 56-68.

Sanger, F., Nicklen, S. \& Coulson, A. R. (1977). DNA sequencing with chain-terminating inhibitors. Proc Natl Acad Sci USA 74, 5463-5467.

Schembri, M., Pallesen, L., Connell, H., Hasty, D. L. \& Klemm, P. (1996). Linker insertion analysis of the FimH adhesin of type 1 fimbriae in an Escherichia coli fim $H$-null background. FEMS Microbiol Lett 137, 257-263.

Sharon, N. (1987). Bacterial lectins, cell-cell recognition and infectious disease. FEBS Lett 217, 145-157.

Sokurenko, E. V., Courtney, H. S., Ohman, D. E., Klemm, P. \& 
Hasty, D. L. (1994). FimH family of type 1 fimbrial adhesins: functional heterogeneity due to minor sequence variations among fim $H$ genes. $J$ Bacteriol 176, 748-755.

Sokurenko, E. V., Courtney, H. S., Maslow, J., Sitonen, A. \& Hasty, D. L. (1995). Quantitative differences in adhesiveness of type 1 fimbriated Escherichia coli due to structural differences in fim $\mathrm{H}$ genes. J Bacteriol 177, 3680-3686.

Sokurenko, E. V., Chesnokova, V., Doyle, R. J. \& Hasty, D. L. (1997). Diversity of Escherichia coli type 1 fimbrial lectin. J Biol Chem 272, 17880-17886.

Stentebjerg-Olesen, B., Pallesen, L., Jensen, L. B., Christiansen, G. \& Klemm, P. (1997). Authentic display of a cholera toxin epitope by chimeric type 1 fimbriae: effects of insert positions and host background. Microbiology 143, 2027-2038.

Thankavel, K., Madison, B., Ikeda, T., Malavia, R., Shah, A. H., Arumugam, P. M. \& Abraham, S. N. (1997). Localization of a domain in the FimH adhesin of Escherichia coli type 1 fimbriae capable of receptor recognition and use of a domain-specific antibody to confer protection against experimental urinary tract infection. J Clin Invest 100, 1123-1136.

Virkola, R., Westerlund, B., Holthofer, H., Parkkinen, J., Kekomaki, M. \& Korhonen, T. K. (1988). Binding characteristics of Escherichia coli adhesins in human urinary bladder. Infect Immun 56, 2615-2622.

Weis, W. I. \& Drickamer, K. (1996). Structural basis of lectin-carbohydrate recognition. Annu Rev Biochem 65, 441-473.

Yamamoto, T., Fujita, K. \& Yokota, T. (1990). Adherence characteristics to human intestinal mucosa of Escherichia coli isolated from patients with diarrhea or urinary tract infections. $J$ Infect Dis 162, 896-908.

Received 5 November 1997; revised 13 February 1998; accepted 23 March 1998. 